\title{
Separation of Fiber Tracts within the Human Cingulum Bundle using Single-Shot STEAM DTI
}

\author{
Alexander Karaus*, Sabine Hofer and Jens Frahm \\ Biomedizinische NMR Forschungs GmbH am Max-Planck-Institut für biophysikalische Chemie, 37070 Göttingen, \\ Germany
}

\begin{abstract}
The cingulum bundle is a complex white matter fiber tract of the human brain that comprises long association fibers, commissural fibers, and various U-fibers. It is located above the corpus callosum and interconnects limbic structures. A decline of the cingulum fiber integrity has been observed in patients with mental disorders such as schizophrenia. In this work a separation of the bi-hemispheric lateral longitudinal striae and selected U-fibers was achieved by fiber tractography of the cingulum bundle based on diffusion tensor imaging at $1.8 \mathrm{~mm}$ isotropic spatial resolution. Anatomic accuracy without susceptibility-induced distortions was ensured by diffusion-weighted single-shot STEAM MRI with 24 gradient directions and $b$ values of 0 and $1000 \mathrm{~s} \mathrm{~mm}^{-2}$. Extending earlier versions, the STEAM sequence combined variable flip angles, centrically reordered phase encoding, partial Fourier encoding, and parallel imaging.
\end{abstract}

Keywords: Human brain, lateral longitudinal stria, U-fibers, diffusion tensor imaging, fiber tractography.

\section{INTRODUCTION}

The primary aim of technical advances in MRI is their use for answering biologic or medical questions. Here, improvements in the design of a diffusion-weighted MRI sequence that does not suffer from susceptibility-induced distortions of the image geometry have allowed for diffusion tensor imaging (DTI) of the human brain at $1.8 \mathrm{~mm}$ isotropic resolution. In a first application fiber tractography of the complex cingulum bundle achieved a separate characterization of individual fiber components.

The cingulum bundle is a thick white matter fiber tract in the cingulate gyrus located at the dorsal surface of the corpus callosum along the midline of both brain hemispheres. Its size and shape results from the complexity of tracts that pass within and through it. Separable tracts not only encircle the corpus callosum as bundles of long association fibers such as the bi-hemispheric lateral longitudinal striae that reciprocally connect frontal, parietal, and temporal areas, but also comprise fibers that radiate into their own gyri (commonly referred to as U-fibers). In a sagittal view the cingulum bundle forms a ring-like belt where the lower anterior ends, projecting into the mamillary bodies, and the lower posterior ends, projecting into the hippocampus, come close together.

Several lines of evidence indicate that the cingulum bundle plays an important role in the regulation of emotional processes $[1,2]$. Abnormal connections between associated cortical structures were found in schizophrenia patients. In line with these observations, cingulotomy was introduced as a treatment for depression and neuropsychiatric illness.

*Address correspondence to this author at the Biomedizinische NMR Forschungs GmbH, 37070 Göttingen, Germany; Tel: +49-551-201-1726; Fax: +49-551-201-1307; Email: akaraus@gwdg.de
However, the heterogeneous composition of the cingulum bundle suggests that any surgical disruption must necessarily involve extensive disconnections among various cortical and subcortical structures. It is therefore of particular importance to resolve the individual fiber systems that build up the cingulum architecture.

The purpose of this work was to identify the lateral longitudinal striae and U-fibers of the cingulum bundle in healthy subjects using DTI-based fiber tractography at adequate spatial resolution and with accurate correspondence to the anatomic MRI from each individual subject. The results are based on anatomic descriptions in post-mortem human and monkey brains [3] and extend previous DTIbased fiber reconstructions of the human cingulum [4-6] by improved spatial resolution, an increased number of gradient directions, and the absence of susceptibility artifacts. The approach is based on the refined development of a diffusionweighted single-shot stimulated echo acquisition mode (STEAM) MRI sequence, which is known to be free from any sensitivity to magnetic field inhomogeneities [7].

To ameliorate the lower signal-to-noise ratio (SNR) of the single-shot STEAM images relative to single-shot EPI scans, the present study employed a novel combination of centrically reordered phase encoding, partial Fourier encoding, and parallel imaging. The latter approach was based on GRAPPA where the separate acquisition of the necessary reference lines led to a full reduction factor of 2 for the actual images. Taken together, the proposed strategy significantly shortened the required train of stimulated echoes and thereby not only reduced the acquisition time of the single-shot images but also enhanced their SNR by allowing for larger readout flip angles due to the lower number of echoes. 


\section{METHODS}

\section{Subjects}

Fifteen human subjects participated in the study, which involved sequence development, experimental optimization of DTI protocols, and final DTI acquisitions of the cingulum bundle (three subjects). None of them had any history of neurologic abnormality. All subjects gave written informed consent before each MRI examination.

\section{Single-Shot STEAM DTI}

MRI studies were conducted on a 3 T MRI system (Tim Trio, Siemens Healthcare, Erlangen, Germany) using a 32channel phased-array head coil. T1-weighted anatomic images were obtained with a radiofrequency-spoiled 3D FLASH MRI sequence with a repetition time of $11 \mathrm{~ms}$, a gradient-echo time of $4.9 \mathrm{~ms}$, and a flip angle of $15^{\circ}$.

DTI acquisitions were performed at $1.8 \mathrm{~mm}$ isotropic resolution using diffusion-weighted single-shot STEAM MRI with 24 diffusion gradient directions and $b$ values of 0 and $1000 \mathrm{~s} \mathrm{~mm}^{-2}$. A schematic drawing of the sequence is shown in Fig. (1a) to identify specific timings and repetition cycles. Briefly, a leading spin-echo (SE) diffusion module gives rise to a diffusion-weighted $\mathrm{SE}$ signal at echo time $\mathrm{TE}_{\mathrm{SE}}$, which replaces the initial $90^{\circ}$ radiofrequency pulse of a single-shot STEAM sequence [8] according to

$90^{\circ}-\mathrm{TE}_{\mathrm{SE}} / 2-180^{\circ}-\mathrm{TE}_{\mathrm{SE}} / 2-\mathrm{SE}-\mathrm{TE}_{\mathrm{STE}} / 2-90^{\circ}-\mathrm{TM}-$ $\left[\alpha-\mathrm{TE}_{\mathrm{STE}} / 2\right]$.

The last (readout) interval in square brackets is repeated with a repetition time $\mathrm{TR}_{\mathrm{STE}}$ in order to generate a sufficiently large number of differently phase-encoded stimulated echoes (STEs) at echo time $\mathrm{TE}_{\mathrm{STE}}$ for covering $\mathrm{k}$ space. It comprises a radiofrequency-spoiled low-flip angle readout pulse, an incremented phase-encoding gradient and corresponding post-acquisition rewinder (to facilitate radiofrequency spoiling of transverse coherences), a frequency-encoding gradient, which is pre-dephased during the initial $\mathrm{TE}_{\mathrm{STE}} / 2$ interval, and an acquisition window centered at the STE. Pre-dephasing of the read gradient also serves to fulfill the stimulated echo condition, which corresponds to full dephasing of all spins prior to the application of the second $90^{\circ}$ radiofrequency pulse of the basic three-pulse sequence [9]. The middle interval TM contains spoiler gradients that preclude the formation of unwanted transverse coherences. All radiofrequency pulses are slice-selective to allow for multi-slice imaging. Putative magnetization crosstalk between neighboring sections is avoided by interleaved acquisition schemes with a long TR for fully relaxed conditions. The outermost repetition cycle in Fig. (1a) includes the desired diffusion gradient directions, the image acquisition without diffusion weighting, and the acquisition of the external reference lines required for parallel imaging.

\section{Experimental Parameters}

The SNR performance of a single-shot STEAM sequence largely depends on the number of STEs required for covering k-space. In a somewhat paradoxic manner - at least when compared to other sequences - a lower number of echoes allows for higher readout flip angles and better SNR, while simultaneously shortening the acquisition time. The present sequence design therefore combines a variety of means to minimize the echo train length ranging from the choice of a rectangular field-of-view (FOV) with the phaseencoding direction matched to the left-right dimension of the subject's head, the use of $6 / 8$ partial Fourier encoding, and the application of parallel imaging based on the GRAPPA technique. The latter approach achieved a full reduction factor of 2 as the 24 reference lines - needed for estimating the coil sensitivities - were obtained in a separate single-shot scan. To further improve the SNR of the reduced echo train, the Cartesian sampling scheme was centrically reordered.

Fig. (1b) illustrates the resulting coverage of k-space as chosen for the present experimental conditions. For DTI of the cingulum bundle, the head was covered by a $191 \mathrm{~mm}$ $(\mathrm{read}) \times 151 \mathrm{~mm}$ (phase) FOV and a $106 \times 84$ matrix yielding $1.8 \mathrm{~mm}$ in-plane resolution. When using partial Fourier encoding with a moderate factor of $6 / 8$, the number of Cartesian Fourier lines reduces from 84 to 63 . The additional implementation of GRAPPA with an acceleration factor of 2 and a separate acquisition of reference lines yields a final number of only 31 lines. As demonstrated in Fig. (1b) the central half of $\mathrm{k}$ space comprises 21 lines (dark gray), which are symmetrically sampled with centric reordering, while only one of the two outer quarters of k-space is linearly sampled by the remaining lines (light gray). For a receiver bandwidth of $220 \mathrm{~Hz} /$ pixel, the actual sequence timings were $\mathrm{TE}_{\mathrm{STE}}=7.08 \mathrm{~ms}$ and $\mathrm{TR}_{\mathrm{STE}}=6.77 \mathrm{~ms}$.

Variable flip angles for the low-flip angle readout pulses were recursively calculated according to

$\alpha_{\mathrm{i}}=\arctan \left[\sin \alpha_{\mathrm{i}+1} \cdot \exp (-\mathrm{TR} / \mathrm{T} 1)\right]$,

which generates equal intensities for the individual STE signals. The consideration of the $\mathrm{T} 1$ decay during acquisition of the echo train (with $\mathrm{T} 1=800 \mathrm{~ms}$ for white matter at $3 \mathrm{~T}$ ) further optimizes the resulting point-spread function and reduces image blurring. The actual flip angles varied from $9^{\circ}$ for the first to $90^{\circ}$ for the $31 \mathrm{st}$ (last) radiofrequency pulse.

The echo time for the diffusion module was $\mathrm{TE}_{\mathrm{SE}}=51.5$ $\mathrm{ms}$, which together with the aforementioned experimental parameters led to a total measurement time of about $290 \mathrm{~ms}$ for the acquisition of an individual diffusion-weighted single-shot image. In order to map the full cingulum bundle 41 oblique sections in AC-PC orientation of $1.8 \mathrm{~mm}$ thickness were acquired without gaps in an interleaved order with $\mathrm{TR}=11.9 \mathrm{~s}$. The resulting measurement time per dataset was 5:10 min, which corresponds to 26 repetition cycles including 24 diffusion-weighted images with different gradient directions, one image without diffusion weighting, and one reference scan for GRAPPA. Noteworthy, if averaging is employed to increase the SNR, it is not necessary to repeat the reference scan. The total measurement time for 3 and 5 acquisitions was 15 and 25 min, respectively.

\section{Fiber Tractography}

Before calculation of the diffusion tensor the diffusionweighted images were interpolated to $0.9 \mathrm{~mm}$ isotropic resolution. Estimates of axonal projections were computed by the tensorlines algorithm [10] and by the fiber assignment by continuous tracking (FACT) algorithm [11] using 9 seed points per image voxel. For a more detailed description of 
1a
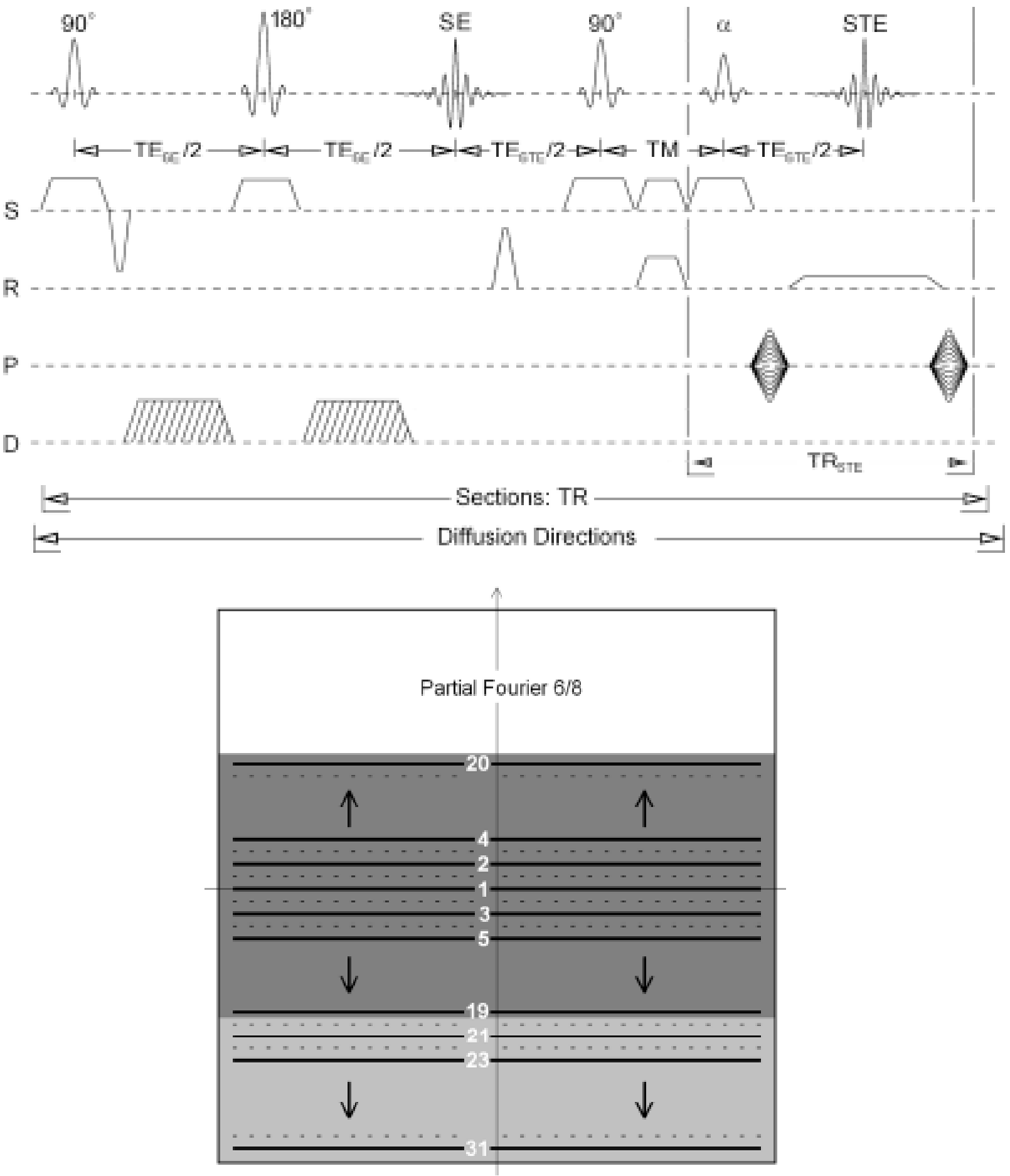

Fig. (1). a: Schematic diagram of the single-shot STEAM DTI sequence, which combines an initial spin-echo (SE) diffusion module (echo time $\mathrm{TE}_{\mathrm{SE}}$ ) with single-shot STEAM MRI (middle interval TM). The sequence generates differently phase-encoded stimulated echoes (STE, echo time $\mathrm{TE}_{\mathrm{STE}}$ ) by repeating the final readout interval (repetition time $\mathrm{TR}_{\mathrm{STE}}$ ) with variable low-flip angle radiofrequency pulses $(\alpha)$. The acquisition of diffusion-weighted images is repeated for multiple sections as well as for different diffusion-encoding gradient directions (repetition time TR). b: Coverage of k-space for Cartesian encoding using centric reordering, 6/8 partial Fourier imaging, and parallel imaging (GRAPPA) with a reduction factor of 2 (reference lines are acquired in a separate scan). The dark shaded area represents the symmetric sampling of the central half of k-space, while the light gray area corresponds to the unilateral sampling of one outer quarter of kspace. The numerical example refers to the parameters chosen here for DTI of the cingulum bundle (for details see text).

the software developed for DTI analysis see [12]. Most importantly, in all cases the selection of a proper region-ofinterest (ROI) was based on a direct comparison with the underlying anatomic structure in each individual subject.
The reconstructions of the different fiber bundles from the cingulum were accomplished with different strategies. (i) The entire cingulum bundle, which comprises more than one fiber system, was obtained with the use of multiple ROIs and 
the tensorlines algorithm restricted to a maximum curvature of $30^{\circ}$. This conservative combination turned out to be most suitable for delineating various long-ranging fiber structures, because the tensorlines algorithm stabilizes the propagation through tensor fields [10]. These properties were confirmed in previous tractography studies of the rhesus monkey brain [13]. (ii) For similar reasons, the lateral longitudinal striae were identified by ROI-to-ROI tracking and the tensorlines algorithm. In coronal sections, the start ROI covered the cingulum in frontal regions closely above the anterior tip of the genu of the corpus callosum, while the target ROI was placed dorso-laterally to the posterior portion of the corpus callosum, where parts of the cingulum bundle travel into the hippocampus. (iii) The reconstruction of U-fibers was based on the FACT algorithm using a single ROI defined in transverse sections. Here, the allowed curvature of the individual U-fibers varied between $40^{\circ}$ and $70^{\circ}$ depending on the anatomic structure and shape of the neighboring gyri. Noteworthy, the results are not very sensitive to the choice of these parameters as the tensorlines algorithm approaches the FACT algorithm for low degrees of stiffness.

To unambiguously separate the tracks belonging to the cingulum from those of the corpus callosum, all commissural fibers that connect cingulum structures between both hemispheres were excluded as these would unavoidably be contaminated by callosal fibers - at least at the presently achievable DTI resolution. It should also be mentioned that the measured DTI maps and reconstructed fiber tracks allowed for a direct digital superposition with the anatomic MRI data without the need for a co-registration or alignment technique.

\section{RESULTS}

The combined implementation of partial Fourier and parallel imaging for diffusion-weighted single-shot STEAM MRI allowed for a reduction of the number of required $\mathrm{k}$ space lines from 84 to 31 for the parameters chosen here. The corresponding reduction of the number of stimulated echoes resulted in a shortening of the image acquisition time from $640 \mathrm{~ms}$ to $290 \mathrm{~ms}$ by more than a factor of two. This property was used to increase the number of sections to enlarge brain coverage. Simultaneously, the higher flip angles for the lower number of stimulated echoes considerably enhanced the resulting SNR. For multiple regions in white matter, the SNR - defined as the mean signal divided by the standard deviation of the noise in an empty part of the image - increased from $26.4 \pm 5.4$ (full Fourier, no parallel imaging) to $73.5 \pm 12.0$ (6/8 partial Fourier, GRAPPA factor 2) by almost a factor of three. This improvement was exploited to increase the spatial resolution.

Fig. (2) shows FA maps of a selected section with contributions from both the cingulum bundle and callosal fibers. The maps differ in the number of acquisitions (1, 3, and 5) and served to decide upon the SNR (and measuring time) required to properly delineate the desired structures of the cingulum bundle. While the FA map based on a single acquisition precludes an unambiguous separation of the cingulum bundle (arrows) from neighboring white matter tracks of the corpus callosum, marked improvements are obtained for 3 and 5 acquisitions. Although the longest acquisition obviously offers the best SNR, detailed fiber tractography of the cingulum bundle based on DTI datasets with only 3 acquisitions yielded very similar results to those obtained with 5 acquisitions. Therefore, all subsequent fiber tracks were derived from 3 acquisitions acquired within a measuring time of $15 \mathrm{~min}$.

In order to assess the reliability of the DTI data for fiber tractography, Fig. (3) shows three color-coded FA maps covering the left-hemispheric cingulum bundle. The maps demonstrate the prevalence of relatively high FA values (green $=0.4-0.6)$ in all regions used for tractography of the longitudinal stria (arrows) and selected U-fibers (dotted spheres). This observation is confirmed by a histogram of FA values derived from the entire left cingulum bundle (Fig. 3 , bottom). In contrast, locations with FA values of 0.1 or below (blue), and therefore low confidence for tractography, refer to ventricular spaces filled with cerebral spinal fluid or to cortical gray matter.

Figs. (4-6) demonstrate the results obtained for the human cingulum bundle (green) in different views and orientations as well as relative to the fibers of the corpus callosum

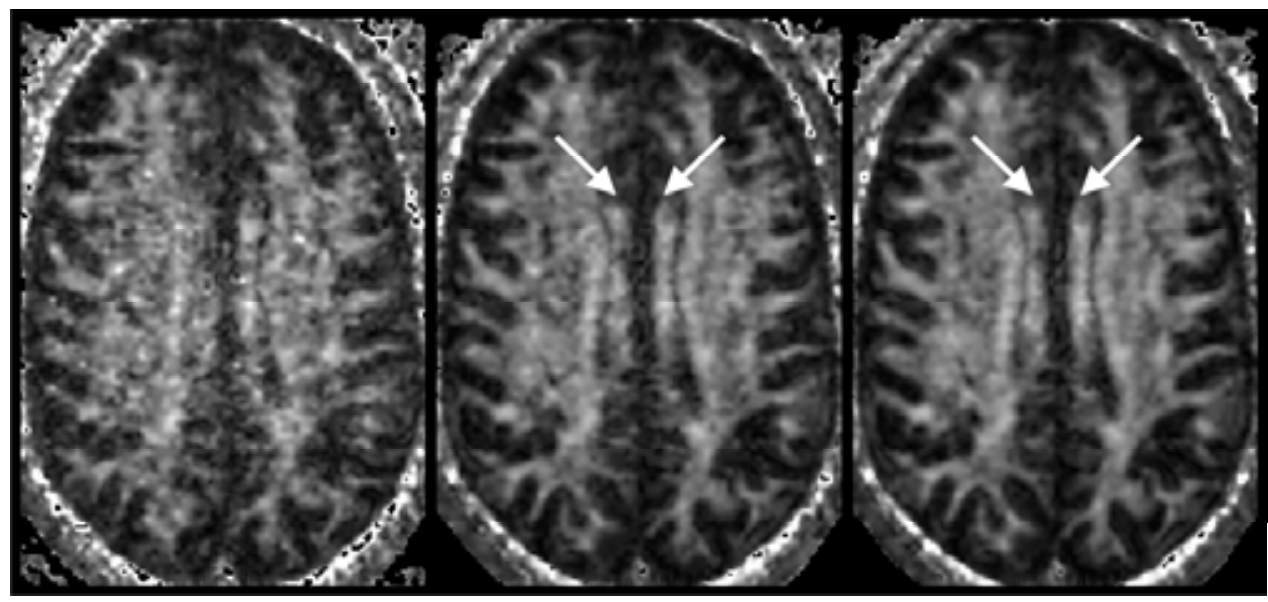

Fig. (2). Fractional anisotropy maps of a selected section covering both the cingulum bundle (arrows) and neighboring callosal fibers at 1.8 mm isotropic resolution (24 gradient directions, $b=0$ and $1000 \mathrm{~s} \mathrm{~mm}^{-2}$ ) for (left) a single acquisition within 5:10 min, (middle) 3 acquisitions within $15 \mathrm{~min}$, and (right) 5 acquisitions within $25 \mathrm{~min}$. 


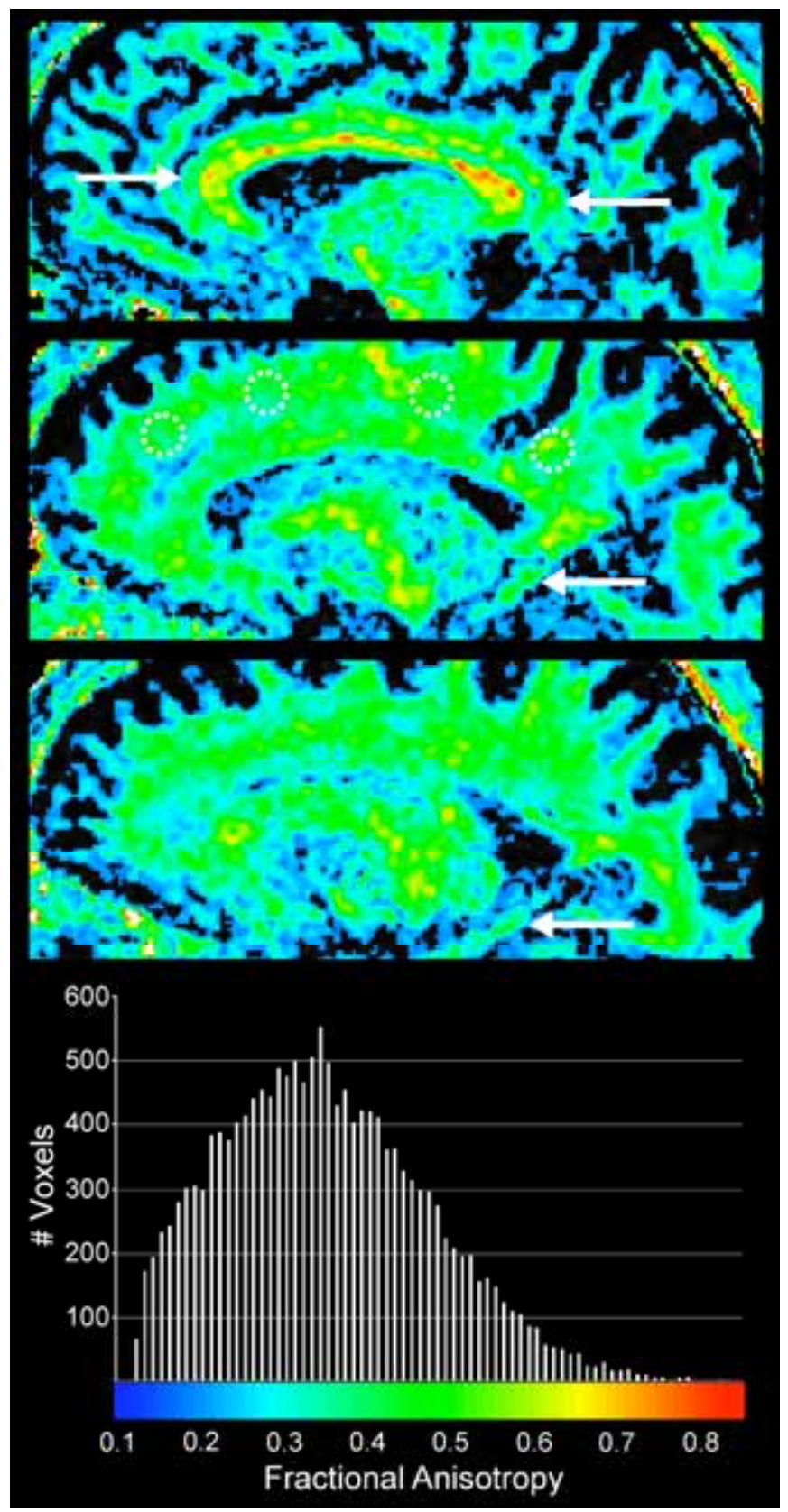

Fig. (3). (Top) Color-coded maps of the fractional anisotropy (FA) representing paramedian sagittal sections of the left hemisphere and (bottom) histogram of FA values derived from the entire left cingulum bundle. The anatomic areas of the longitudinal stria (arrows) or Ufibers (dotted spheres) are characterized by relatively high FA values (green $=0.4-0.6$ ), which is quantitatively confirmed by the corresponding FA histogram. DTI was performed at $1.8 \mathrm{~mm}$ isotropic resolution (41 sections, 24 gradient directions, $b=0$ and $1000 \mathrm{~s}$ mm ${ }^{-2}$ ) using 3 acquisitions (measuring time $15 \mathrm{~min}$ ). For further details see text.

(yellow). The data originate from a single subject, but similar reconstructions of the longitudinal striae and U-fibers with respect to size and shape were obtained from all three subjects studied with an identical DTI protocol. Because the respective fiber systems are partially overlapping, they are separately depicted in sagittal views in Fig. (4). The lateral longitudinal striae (red) as one of the longest fiber bundles runs from the frontal lobe to the parahippocampal gyrus in both hemispheres: it is located above the corpus callosum, stretches around the cingulate gyrus, and revolves around the splenium by almost 180 degrees. The much shorter U-fibers (blue) that connect directly neighboring structures enter and leave the cingulum bundle along its entire extension bilaterally. The relative position of the corpus callosum, overall cingulum bundle, lateral longitudinal striae, and local U-fibers is clearly visualized in coronal (two-dimensional) representations. As shown for a coronal section in the left part of Fig. (5), the lateral longitudinal striae are fully embedded within other cingulum fibers, which - together with the U-fibers - are enclosed by fibers of the corpus callosum. Accordingly, in the top view in the right part of Fig. (5), the fibers of the corpus callosum largely conceal those of the cingulum bundle. Finally, Fig. (6) represents three-dimensional views of the cingulum fibers, the- 


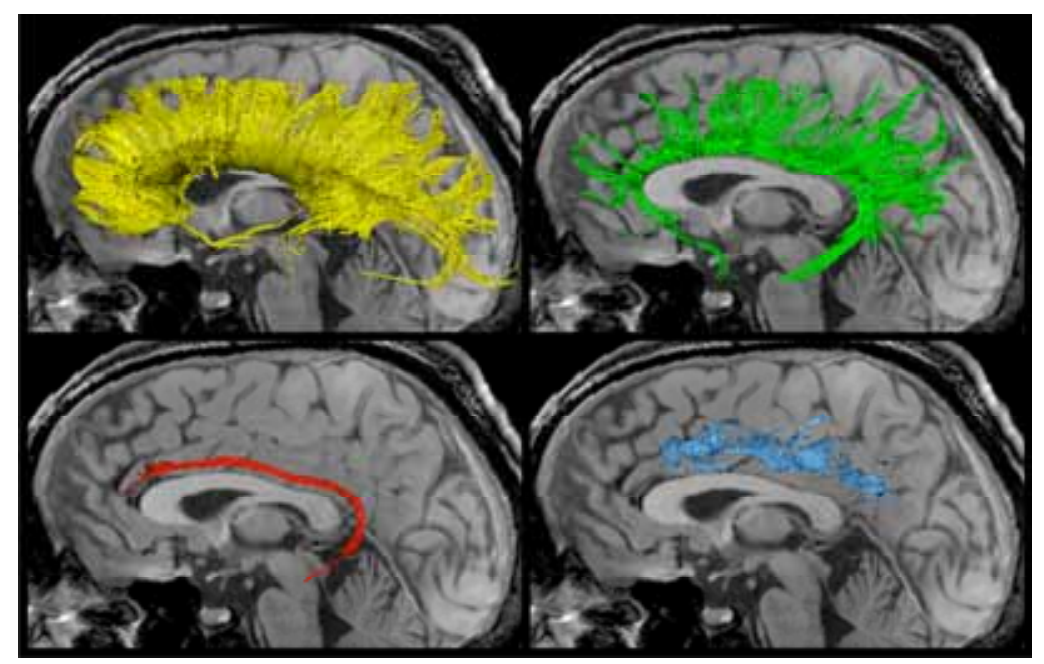

Fig. (4). Sagittal three-dimensional views of fiber reconstructions from the corpus callosum (yellow), cingulum bundle (green), lateral longitudinal striae (red), and associated U-fibers (blue) relative to the subject's individual anatomy. For experimental parameters (see Fig. 3).

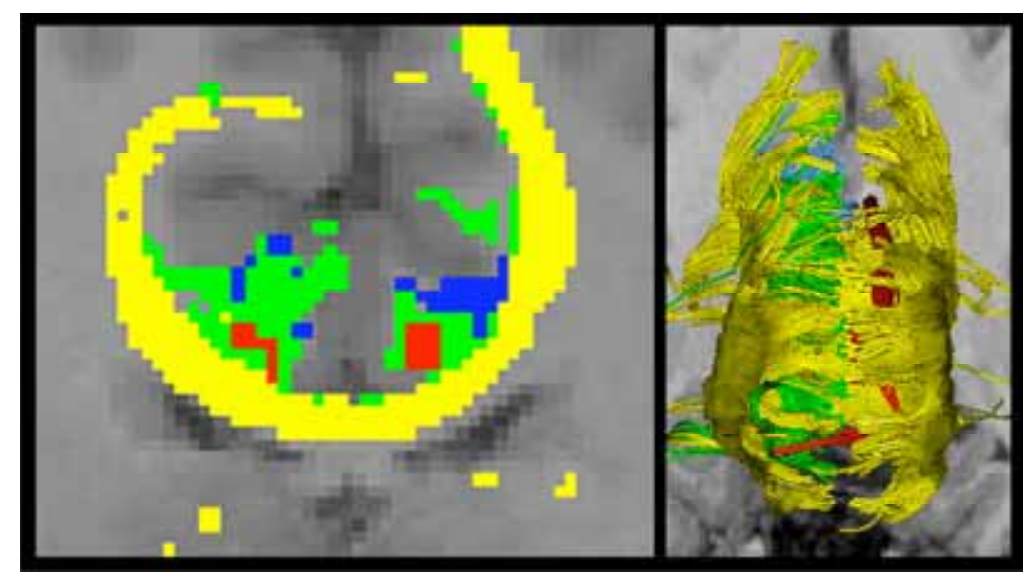

Fig. (5). Fiber reconstructions from the corpus callosum (yellow), cingulum bundle (green), lateral longitudinal striae (red), and local Ufibers (blue) in (left) a coronal two-dimensional representation and (right) a three-dimensional top view. For experimental parameters (see Fig. 3).

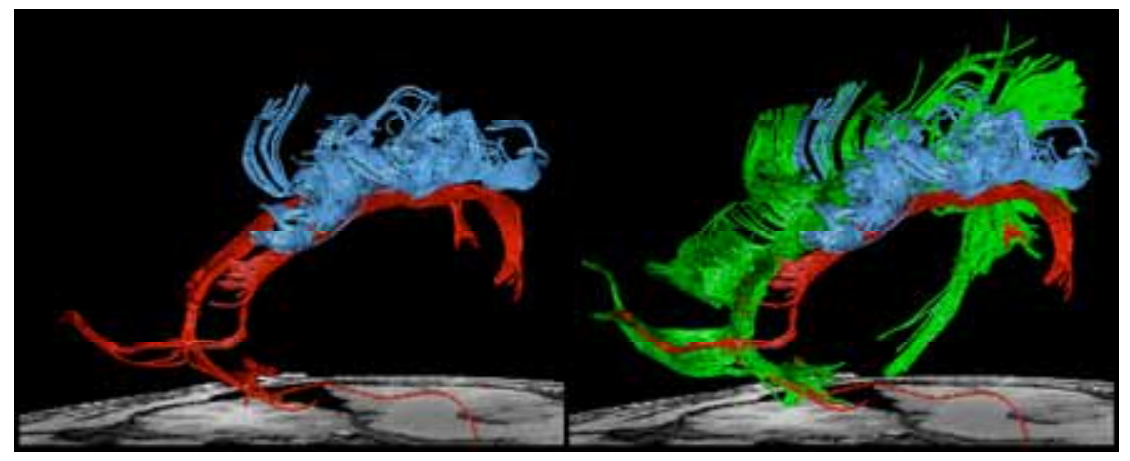

Fig. (6). Oblique three-dimensional views of fiber reconstructions from the right cingulum bundle (green), right and left lateral longitudinal striae (red), and selected U-fibers (blue). For experimental parameters (see Fig. 3).

bihemispheric lateral longitudinal striae, and several U-fibers without the surrounding transcallosal tracks.

\section{DISCUSSION}

Geometrically correct DTI-based fiber tractography of the cingulum bundle in healthy human subjects resolved important substructures in a manner fully consistent with the corresponding individual anatomy as well as in agreement with existing literature data [3]. In particular, this work identified the bi-hemispheric association fibers termed lateral longitudinal striae and several U-fibers. The possibility to distinguish individual tracks that enter and leave the 
cingulum will aid to the neurobiology of mental disorders that involve a degeneration of cingulum fibers. For example, in schizophrenia this refers to the assessment of putative deficiencies not only in the direct communication between the frontal and temporal lobe, but also in the inter- and intrahemispheric communication between various cortical and subcortical structures of the limbic system.

Although major portions of the human cingulum have previously been reconstructed by DTI-based fiber tractography [4-6], none of these reports separately identified subcomponents of the cingulum such as the lateral longitudinal striae by individual fiber tracking and in direct correspondence to the individual anatomy. In a technical sense, and with respect to clinically oriented applications, further improvements are warranted. This particularly applies to a reduction of the total measurement time and probably more important - to a better spatial resolution to reduce partial volume effects and ameliorate some of the limitations of state-of-the-art DTI. In either case, the crucial factor remains the achievable SNR, which for STEAM MRI may be enhanced with the use of multi-shot sequences that lead to even shorter echo trains than used here. The approach has recently been demonstrated to considerably improve black-blood imaging of the human heart [14]. The corresponding development of a segmented diffusionweighted STEAM MRI technique that solves the problem of motion-induced phase differences between individually acquired parts of k-space appears promising [15].

\section{ACKNOWLEDGEMENTS}

We thank Professor Wolfgang Seeger, Hannover, for stimulating discussions about the fine structure of the cingulum and its clinical relevance in mental disorders. We acknowledge financial support by the German Ministry for Education and Research (BMBF) via the Bernstein Center for Computational Neuroscience (BCCN) Göttingen under Grant No. 01GQ0431.

\section{ABBREVIATIONS}

$\begin{array}{ll}\text { DTI } & =\text { Diffusion tensor imaging } \\ \text { FA } & =\text { Fractional anisotropy } \\ \text { FACT } & =\text { Fiber assignment by continuous tracking } \\ \text { FOV } & =\text { Field-of-view } \\ \text { ROI } & =\text { Region-of-interest }\end{array}$
SNR $=$ Signal-to-noise ratio
SE $=$ Spin echo
STE $=$ Stimulated echo
STEAM $=$ Stimulated echo acquisition mode

\section{REFERENCES}

[1] Nestor PG, Kubicki M, Spencer KM, Niznikiewicz M, McCarley $\mathrm{RW}$, Shenton ME. Attentional networks and cingulum bundle in chronic schizophrenia. Schizophr Res 2007; 90: 308-15.

[2] Catheline G, Periot O, Amirault M, et al. Distinctive alterations of the cingulum bundle during aging and Alzheimer's disease. Neurobiol Aging 2008; [Epub ahead of print]

[3] Schmahmann JD, Pandya DN. Fiber Pathways of the Brain. Oxford: Oxford University Press 2006

[4] Catani M, Howard RJ, Pajevic S, Jones DK. Virtual in vivo interactive dissection of white matter fasciculi in the human brain. Neuroimage 2002; 17: 77-94.

[5] Catani M, de Schotten MT. A diffusion tensor imaging tractography atlas for virtual in vivo dissections. Cortex 2008; 44: 1105-32.

[6] Lawes INC, Barrick TR, Murugam V, et al. Atlas-based segmentation of white matter tracts of the human brain using diffusion tensor tractography and comparison with classical dissection. Neuroimage 2008; 39: 62-79.

[7] Nolte UG, Finsterbusch J, Frahm J. Rapid isotropic diffusion mapping without susceptibility artifacts. Whole brain studies using diffusion-weighted single-shot STEAM MR imaging. Magn Reson Med 2000; 44: 731-6.

[8] Frahm J, Haase A, Matthaei D, Merboldt KD, Hänicke W. Rapid NMR imaging using stimulated echoes. J Magn Reson 1985; 65: 130-5.

[9] Frahm J, Merboldt KD, Hänicke W, Haase A. Stimulated echo imaging. J Magn Reson 1985; 64: 81-93.

[10] Weinstein D, Kindlmann G, Lundberg E. Tensorlines: advectiondiffusion based propagation through diffusion tensor fields. Proc IEEE Vis 1999; 249-53.

[11] Mori S, Crain BJ, Chacko P, van Zijl PC. Three-dimensional tracking of axonal projections in the brain by magnetic resonance imaging. Ann Neurol 1999; 45: 265-9.

[12] Küntzel M. Parallele datenakquisition zur beschleunigung diffusionsgewichteter kernspintomographie mit stimulierten Echos Parallel data acquisition for diffusion-weighted MRI using stimulated echoes. Dissertation. Göttingen: Georg-AugustUniversität 2007.

[13] Hofer S, Frahm J. In vivo mapping of fiber pathways in the rhesus monkey brain. Open Med Imaging J 2008; 2: 32-41.

[14] Karaus A, Merboldt KD, Graessner J, Frahm J. Black-blood imaging of the human heart using rapid stimulated echo acquisition mode (STEAM) MRI. J Magn Reson Imaging 2007; 26: 1666-71.

[15] Uecker M, Karaus A, Frahm J. Inverse reconstruction method for segmented multi-shot diffusion-weighted MRI with multiple coils. Magn Reson Med 2009; doi: 10-1002/mrm.22126, Sep 24. [Epub ahead of print]. 